
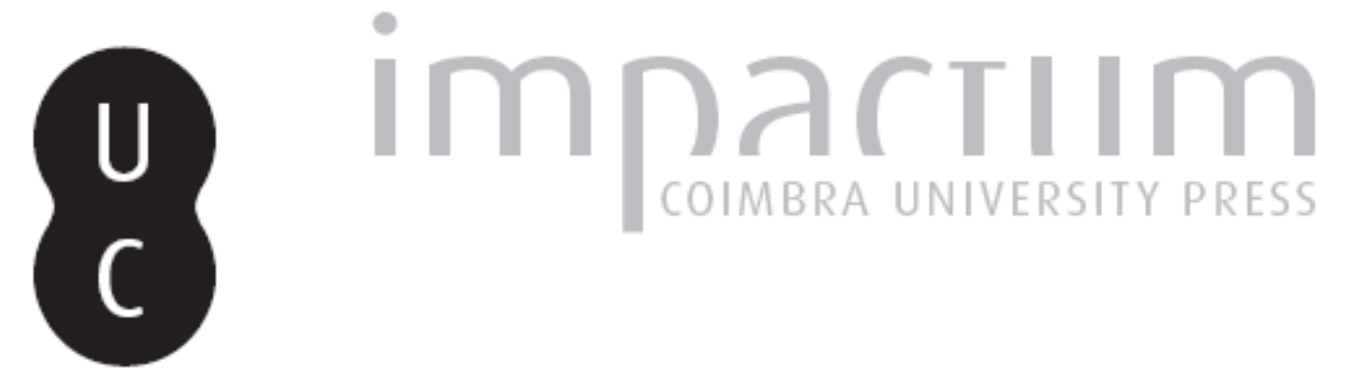

\title{
P. Ricoeur sous le signe d'Aristote: du tragique de l'action à la sagesse pratique
}

\section{Autor(es): $\quad$ Portocarrero, Maria Luísa}
Publicado por: Faculdade de Letras da Universidade de Coimbra, Instituto de Estudos Filosóficos

URL persistente:

URI:http://hdl.handle.net/10316.2/29581

DOI:

DOI:http://dx.doi.org/10.14195/0872-0851_40_6

Accessed : $\quad$ 26-Apr-2023 14:18:58

A navegação consulta e descarregamento dos títulos inseridos nas Bibliotecas Digitais UC Digitalis, UC Pombalina e UC Impactum, pressupõem a aceitação plena e sem reservas dos Termos e Condições de Uso destas Bibliotecas Digitais, disponíveis em https://digitalis.uc.pt/pt-pt/termos.

Conforme exposto nos referidos Termos e Condições de Uso, o descarregamento de títulos de acesso restrito requer uma licença válida de autorização devendo o utilizador aceder ao(s) documento(s) a partir de um endereço de IP da instituição detentora da supramencionada licença.

Ao utilizador é apenas permitido o descarregamento para uso pessoal, pelo que o emprego do(s) título(s) descarregado(s) para outro fim, designadamente comercial, carece de autorização do respetivo autor ou editor da obra.

Na medida em que todas as obras da UC Digitalis se encontram protegidas pelo Código do Direito de Autor e Direitos Conexos e demais legislação aplicável, toda a cópia, parcial ou total, deste documento, nos casos em que é legalmente admitida, deverá conter ou fazer-se acompanhar por este aviso.

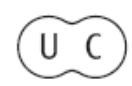




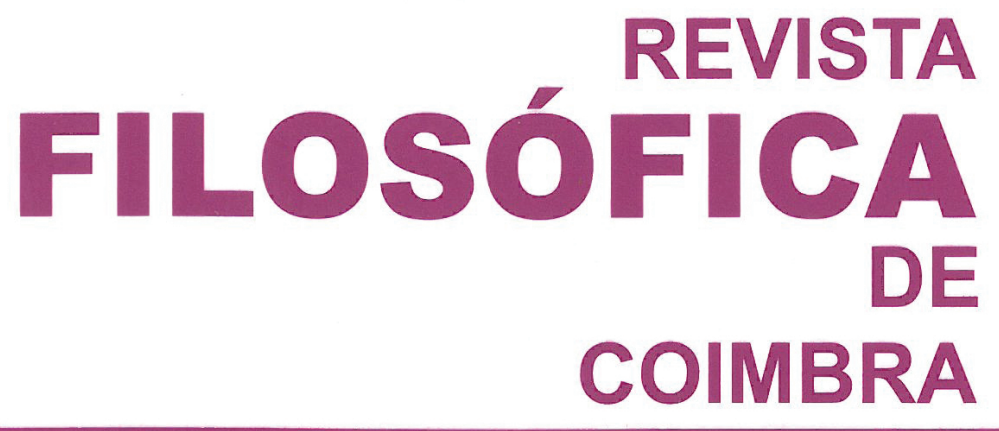

vol. 20 - número 40 - outubro 2011

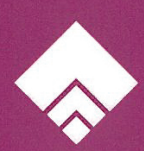




\title{
P. RICOEUR SOUS LE SIGNE D'ARISTOTE: DU TRAGIQUE DE L'ACTION À LA SAGESSE PRATIQUE
}

\author{
MARIA LUÍSA PORTOCARRERO ${ }^{1}$
}

Resumo: Este artigo procura explicar como o filósofo francês P. Ricoeur actualiza, graças ao seu conceito de sabedoria prática, a mensagem fundamental da ética aristotélica. Parte-se da importância que Ricoeur atribui à formação da consciência por relação à norma universal e problematiza-se, recorrendo à tragédia Antígona, a aplicação puramente deontológica da norma. Se para a ética prática contemporânea o legado do imperativo categórico kantiano é irrefutável, é o conflito causado pelo trajecto descendente da ética, o da norma em direcção à situação concreta, que exige uma sabedoria prática, de tipo aristotélico, que saiba inventar as condutas que, traindo a norma o menos possível, é capaz de reconhecer o carácter insubstituível da pessoa singular.

Palavras clave: Ricoeur, Aristóteles, ética prática, norma.

Résumé: Cette communication cherche à expliciter la façon dont le philosophe français $\mathrm{P}$. Ricoeur actualise, grâce à son concept de sagesse pratique, le message fondamental de l'éthique aristotélicienne. On part pour cela de l'importance que Ricoeur attribue à la formation de la conscience par relation à la norme universelle et on questionne, en recourant à la tragédie Antigone, l'application purement déontologique de la norme. Si, pour l'éthique pratique contemporaine, l'héritage kantien de l'impératif catégorique est irréfutable, c'est le conflit provoqué par le trajet descendant de l'éthique, celui de la norme à la situation concrète, qui requiert une sagesse pratique, de type aristotélicien, qui sache inventer les conduites qui, tout en trahissant le moins possible la norme, sont capables de reconnaître le caractère irremplaçable de la personne singulière.

Mots-clefs: Ricoeur, Aristote, éthique pratique, norme.

${ }^{1}$ Departamento de Filosofia, Comunicação e Informação (Faculdade de Letras da Universidade de Coimbra). 
Le philosophe P. Ricoeur prête une attention toute particulière au rôle toujours joué par la norme universelle et contraignante dans la configuration de l'identité humaine. C'est notamment dans l'œuvre Soi-même comme un autre ${ }^{2}$ et dans les deux Justes ${ }^{3}$ que ses analyses sont centrées sur la dimension éthique, narrative et institutionnelle de la formation de notre identité personnelle. L'accent y est mis sur la capacité qu'a l'homme de s'inscrire dans l'espace public des règles et des normes communes. Donc, sur sa capacité d'entrer dans le cercle de la reconnaissance de la liberté de l'autre, phénomène qui implique la réciprocité et aussi la valeur des institutions dans l'aventure de concrétisation du désir humain de bonheur.

Ayant dédié toute sa philosophie à la pensée de l'homme réel, toujours déjà lancé dans le monde temporel de l'interaction, c'est pour une rationalité capable de dire «le désir d'une vie bonne avec des autres dans des institutions justes» et les conflits éthiques qui ourdissent la condition plurielle de l'homme que Ricoeur travaille à sa philosophie éthique. Héritier de la tradition réflexive et de son exigence de rigueur et profondément influencé par la Phénoménologie, dans son anthropologie du vécu temporel, Ricoeur est profondément marqué, dans sa tournure philosophique, par la vertu du conflit (ontologique et éthique). La nouvelle rationalité doit donc se situer, dorénavant, dans une position médiatrice entre désir et norme, prenant alors clairement ses distances vis-à-vis de la rationalité abstraite et solipsiste des philosophies du Cogito. C'est pour cette raison que la relation de la conscience à la norme universelle et contraignante, autrement dit aux principes du permis et du défendu, devient un noyau déterminant des réflexions éthiques de notre philosophe.

Mais ajoutons: Ricoeur ne veut pas partir, à la manière de Kant, d'une opposition frontale entre la loi (immuable, contraignante et universelle) et la conscience quotidienne désirante, donc considérée comme variable, circonstancielle, passionnelle et éminemment subjective ${ }^{4}$. Ce type de dialectique consacre d'après Ricoeur : a) ou bien une morale de l'obligation, purement formelle, qui oublie l'enracinement de l'éthique dans la praxis, parce qu'elle ne valorise que le rôle de la norme et ne prête aucune attention aux situations affectives et concrètes de l'agir; tel est le cas des morales déontologiques; b) ou bien, à l'inverse, une casuistique pure

\footnotetext{
2 P. RICOEUR (1990).

${ }^{3}$ Cf. P. RICOEUR (1995) ; P. RICOEUR (2001).

${ }^{4}$ P. RICOEUR (1995 : 209).
} 
qui rend relatif le rôle de toutes les règles. Le philosophe sait que ces deux modèles gouvernent le panorama éthique contemporain et il veut, au contraire, «construire un modèle plausible de corrélation entre les termes d'une alternative ${ }^{5} \gg$, qu'il considère profondément ruineuse, parfois même tragique.

Pour nous éclairer sur les effets nocifs du manque de médiation entre norme universelle et casuistique de l'affectivité, notre philosophe recourt à l'histoire de l'humanité elle-même et attire notre attention sur le tragique de l'action, exemplifié de façon dramatique par l'Antigone de Sophocle. «Afin de restituer au conflit la place que toutes les analyses conduites jusqu'ici ont évité de lui accorder, il nous a paru approprié de faire entendre une autre voix que celle de la philosophie - même morale ou pratique -, une des voix de la non-philosophie : celle de la tragédie grecque. De cette irruption intempestive, nous attendons le choc susceptible d'éveiller notre méfiance à $l^{\prime}$ encontre non seulement des illusions du cœur, mais aussi des illusions nées de 1'hubris de la raison pratique elle-même» ${ }^{6}$.

Cette tragédie est bien l'exemple vivant des excès provoqués par le conflit entre la rigidité de la norme et l'inflexibilité dans la transgression: «Si, effectivement, j'ai choisi Antigone, c'est parce que cette tragédie dit quelque chose d'unique en ce qui concerne le caractère irréductible du conflit dans la vie morale et, en outre, esquisse une sagesse, la sagesse tragique dont parlait Jaspers - capable de nous orienter dans les conflits (...). Si la tragédie Antigone peut encore nous servir d'enseignement, c'est parce que le propre contenu du conflit - en dépit du caractère perdu et non répétable du fond mythique dont il émerge et de l'environnement festif qui entoure la célébration du spectacle - a conservé une permanence ineffaçable ${ }^{7} \gg$.

Interrogeons nous donc: quelle est la raison pour laquelle le drame d'Antigone nous émeut-il toujours aujourd'hui? Est-il, ou non, un témoignage historique de l'inviolable dignité de l'humain, quand bien même Polynice est réduit à l'état de cadavre? Rappelons l'histoire : la jeune Antigone se refuse, dans la tragédie de Sophocle du même nom, à laisser le corps de son frère Polynice, accusé de traître par Créon, être réduit à pâture pour chiens et oiseaux. Elle réclame ainsi, de par son action même et en mettant en risque sa vie, une sépulture pour son frère, ce qui veut dire qu'elle agit dans le but de certifier et d'honorer l'appartenance fondamentale de son frère mort à la communauté humaine, celle qui est

\footnotetext{
5 P. RICOEUR (1995: 209).

${ }^{6}$ P. RICOEUR (1990: 281).

7 P. RICOEUR (1990 : 283).
} 
caractérisée par les rites de sépulture et les symboles qui assurent l'inviolable dignité de l'humain, même réduit à l'état de cadavre.

La question est donc celle- ci: Antigone a-t-elle toujours aujourd'hui une valeur éthique qui puisse faire réfléchir les philosophes de l'éthique? Et quel est donc son message fondamental? Selon Ricoeur, ladite tragédie touche le fond agonistique de l'épreuve humaine «où s'affrontent, interminablement, l'homme et la femme, la vieillesse et la jeunesse, la société et l'individu, les vivants et les morts, l'humain et le divin ${ }^{8}$ ». Par son intermédiaire, nous faisons, effectivement, le dur apprentissage de la condition finie, celle du choix et du conflit, et c'est encore grâce à elle que nous sommes menés à réfléchir dans le but de refuser le caractère non négociable des conflits axiologiques. Ricoeur sait bien sûr que la tragédie, en tant que poésie, ne procède pas conceptuellement. C'est la succession des Odes lyriques du chœur et les paroles de Hémon et de Tirésias qui produisent une conversion du regard que l'éthique devra développer dans son propre discours. En effet, ce qui se passe chez Antigone, c'est que, lorsqu'elle invoque les lois non écrites pour fonder sa conviction intime, celle de donner sépulture à son frère, elle a dénoncé quelque chose que, la plupart du temps, nous oublions: le caractère inviolable de la dignité humaine et la nature humaine, trop humaine, de toute l'institution. C'est quand même l'environnement institutionnel qui assure les conditions de la dignité.

Antigone a donc dénoncé, par sa mort tragique, les limites du légalisme, c'est-à-dire, celle des normes trop rigides qui ne permettent pas que l'agent moral soit irrigué par le vœu de répondre à une attente, voire à une requête venue d'autrui. Elle a donc exemplifié l'urgence d'une sagesse du jugement en situation.

\section{II}

Quelle instruction donc nous retenons de cette histoire ? Un appel à to fronein, «un appel à bien délibérer traverse obstinément la pièce» 9 . D'où l'importance inaliénable de l'éthique d'Aristote dans ce trajet de la norme à la délibération. En effet, mettant en scène les conflits insolubles de la condition humaine, la tragédie, «après avoir désorienté le regard, condamne l'homme de la praxis à réorienter l'action à ses propres risques et frais, dans le sens d'une sagesse pratique en situation qui réponde le

${ }^{8}$ P. RICOEUR (1990: 283).

9 P. RICOEUR (1990 : 287). 
mieux à la sagesse tragique» ${ }^{10}$. C'est donc la place inévitable du conflit dans la vie morale que Ricoeur veut penser avec sa sagesse pratique en faisant constamment recours à la prudence d'Aristote.

Retenons donc: si en effet les normes sont nécessaires et accompagnent la formation de la conscience, et dans ce sens Ricoeur est kantien; si elles «appellent pour vis-à-vis un être capable d'entrer dans un ordre symbolique pratique, c'est-à-dire de reconnaître dans les normes une prétention légitime à régler les conduites ${ }^{11}{ }^{1}$, elles n'existent pas pour leur propre gloire, mais pour être appliquées. Ce qui veut dire qu'elles sont des schémas d'action et qu'elles deviennent trop rigides quand elles oublient la valeur du jugement moral en situation, propre d'une sagesse pratique, qui sache évaluer en détail et en terme de pluralité les tenants et les aboutissants d'un conflit éthique. Pour Ricoeur, il faudra toujours montrer de quelle manière les conflits suscités par le formalisme, étroitement lié au moment déontologique, nous reportent de la morale à l'éthique, mais à une éthique enrichie par le passage par la norme inscrite dans le jugement moral en situation ${ }^{12}$.

De par ses réflexions sur Antigone, P. Ricoeur nous mène donc à une longue méditation sur la place inévitable du conflit dans la vie morale et sur le rôle joué par les agents moraux, quand ils sont au service de grandeurs spirituelles qui les dépassent et, qui sont, parfois, des sources de malheur. Notamment quand la norme est interprétée de façon tellement rigide qu'elle oublie l'altérité des personnes, inhérente à l'idée même de pluralité humaine, et la conviction sert de motif à une transgression presque fanatique.

Ce qui est donc en jeu, à partir du message qui nous a été laissé par la tragédie Antigone, c'est un appel à bien délibérer qui, comme nous le voyons, ne se résume pas à appliquer la norme, sans appel ni offense, et d'autant moins à la cristallisation dans la conviction; en d'autres mots, ici sont en jeu les limites de l'éthique déontologique et de la casuistique pure. Ces deux types d'éthique s'affrontent, nous le savons, dans le panorama contemporain. Mais ce qui est fondamentalement au coeur du débat, dans cette réflexion de Ricoeur, c'est son héritage kantien, philosophe moderne qui a consacré de façon absolue la morale déontologique ou morale de l'obligation (sans transgression) et dont l'effet historique a dominé non seulement la philosophie continentale, mais aussi le Droit.

Kant, nous le savons, était fondamentalement préoccupé, à son épo-

${ }^{10}$ P. RICOEUR $(1990: 288)$.
${ }^{11}$ P.RICOEUR $(2001: 58)$.
${ }^{12}$ Cf.,P. RICOEUR $(1990: 317-318)$. 
que, par le trajet de confirmation de l'universalité d'une norme morale, quelle qu'elle soit. C'est à cause de cela qu'il a oublié le problème des conflits dans l'application concrète des normes. Or, c'est dans ce second trajet, descendant, de l'appréciation de la situation concrète par la norme, moment où les personnes en jeu, en situation, exigent d'être reconnues et non sacrifiées, qu'apparaît le conflit ${ }^{13}$, que le philosophe de Königsberg n'a jamais reconnu. Le problème éthique de Kant avait des racines fortement épistémologiques, d'où le fait que sa morale soit caractérisée par une stratégie d'épuration de la conscience humaine, c'est-à-dire, par un processus de distanciation progressive des situations particulières, de façon à ce que l'on puisse atteindre la bonne volonté, sans conditions. Seule cette volonté est législatrice d'elle-même ou autonome, ce qui veut dire qu'elle est celle qui sait se donner à elle-même la loi universelle, parce qu'elle a déjà réussi à se libérer de l'esclavage de désirs, de sentiments et de penchants. D'où le célèbre impératif: agis de telle façon que la loi universelle soit la norme de ton action.

Ricoeur, notons-le, bien que critique vis-à-vis de l'universalisme formel, rend pleinement justice à Kant, car il reconnaît que le principe de l'autonomie a toute raison d'être: il apparaît, effectivement comme étant le seul moyen de résistance contre les pressions issues des inclinations somatiques de chacun, contre celles du monde de l'opinion et de l'influence des autres. Il valorise donc Kant: c'est que, au cours de la formation de l'identité humaine, la dimension des normes communes et des structures éthiques qui guident les rapports intersubjectifs est absolument indispensable. La morale kantienne peut et doit ainsi être considérée, dans ses grandes lignes, comme une recension exacte de l'expérience morale commune, selon laquelle ne peuvent être tenues pour obligatoires uniquement les maximes de l'action qui satisfont le test de l'universalisation. Cependant, notre philosophe pense aussi qu'il n'est pas nécessaire d'apprécier, à la façon de Kant, le devoir comme un ennemi du désir de bonheur, ni de réduire l'universalisation à la non contradiction, présupposé qui domine le formalisme de Kant et qui nous donne une idée très pauvre de la cohérence d'un système moral ${ }^{14}$.

Pour dépasser ce type de formalisme et nous montrer que l'idée elle-même d'obligation morale prend ses racines, avant Kant, dans le désir d'une vie heureuse, qui caractérisait déjà la conscience morale chez Aristote, Ricoeur effectue une méditation ${ }^{15}$ sur la constitution éthique,

\footnotetext{
13 P. RICOEUR (1990: 307).

14 P. RICOEUR (1990: 321).

15 P. RICOEUR (1992: 203-221).
} 
dialogique et temporelle de la personne humaine. Et il découvre toute une «dialectique plus radicale de l'éthos, susceptible de fournir un fil conducteur dans l'exploration des autres couches de la constitution de la personne ». (...) «Je propose la définition suivante de l'ethos : souhait d'une vie accomplie - avec et pour les autres - dans des institutions justes. Ces trois termes me paraissent également importants pour la constitution éthique de la personne (...). Souhait d'une vie accomplie : en inscrivant ainsi l'éthique dans la profondeur du désir, on souligne son caractère de souhait, d'optatif, antérieur à tout impératif ${ }^{16}$ ».

Par le biais de cette réflexion Ricoeur cherche, encore, à identifier les niveaux de la norme et de la transgression qui sont simultanément présents dans l'émergence de la conscience et de l'interaction humaine. Le but vraiment poursuivi est celui de nous faire comprendre comment la conscience morale qui, justement, représente la différence entre l'homme concret, l'animal et le robot, naît - avant toute relation avec la norme-, dans la lignée aristotélicienne, du désir d'une vie heureuse avec d'autres dans des institutions justes: «(...) La formule complète serait: Ah puisséje vivre bien sous l'horizon d'une vie accomplie et, en ce sens, heureux! L'élément éthique de ce désir ou vœu peut être exprimé par la notion d'estime de $\operatorname{soi}^{17}$ »).

Sans cette dimension de l'estime de soi, une quelconque éthique est impossible et Ricoeur nous prévient encore: l'estime de soi, dont il s'agit ici, n'a rien d'égoïsme ou de solipsisme, étant donné que le terme soi est utilisé pour éviter toute réduction à un je centré sur lui-même ${ }^{18}$. Il correspond, au contraire, à l'élément réflexif de toutes les personnes grammaticales, outre le fait que l'estime de soi n'est rien sans l'intimation éthique plus profonde, celle de la réciprocité ou de la reconnaissance de l'autre. «L'autre, mon semblable, tel est le vote de l'éthique en ce qui concerne la relation entre l'estime de soi et la sollicitude ${ }^{19} \gg$. C'est bien dans l'amitié que la ressemblance et la reconnaissance se réalisent le mieux, mais il ne faut pas oublier qu'il existe aussi une figure de l'autre, celui que je rencontre aussi mais qui n'est pas mon ami. Il s'agit du tiers, celui auquel je fais face au sein des institutions: «(...) la personne qui est en face, sans visage, le tout à chacun d'une distribution juste ${ }^{20}$ ». L'autre impliqué dans l'éthos est toujours double, l'ami et le «tout à chacun» de
16 P. RICOEUR (1992: 204).
17 P. RICOEUR (1992 : 204-205).
18 P. RICOEUR (1992: 205).
19 P. RICOEUR (1990: 205).
20 P. RICOEUR (1992 : 206). 
l'idée de justice. J'évoque ici, nous dit Ricoeur, «l'analyse aristotélicienne de la justice qui se prolonge jusqu'aux traités médiévaux. (...). C'est à ce problème de la justice dans un partage inégal que s'applique exactement l'idée de justice distributive depuis Aristote ${ }^{21} \gg$.

Ainsi, toute la conscience est absolument solidaire de l'apparition de l'autre et la loi qui existe pour être appliquée mais qui, pour l'être, exige, dans la lignée de la prudence aristotélicienne, sa traduction dans la situation singulière de sa concrétisation. La triple constitution de l'éthos, attention prêtée à soi-même, attention prêtée à l'autre et à l'institution, me montre en effet que le désir éthique d'une vie heureuse, lui-même, sans l'institution ni la loi, se désagrège, se réduit à un désir illusoire, étant donné que le mal et la violence existent effectivement. Cependant, on doit montrer à Kant que la loi a toujours des bords peu clairs, qu'elle n'est rien en elle-même, c'est-à-dire, sans la médiation de la situation qui réclame justice. Autrement dit, si Ricoeur est d'accord avec les intuitions de Kant, en ce qui concerne la dimension universelle et contraignante des normes, il n'oublie toutefois pas non plus les sages enseignements d'Antigone - nouvelle orientation du regard éthique en vue d'une conciliation par le renoncement, par le pardon et par la reconnaissance ${ }^{22}$. Et, étant fidèle à son grand maître G. Marcel, il oppose à Kant cette maxime: «c'est à l'autre que je veux être fidèle» ${ }^{23}$. Il pose donc la question: les personnes seraient-elles vraiment reconnues quand le respect se dirige seulement et fondamentalement à la loi? C'est de ce doute que dérive son propos d'une corrélation herméneutique et délibérative entre loi et conscience, appelée la sagesse pratique. La sagesse pratique, nous dit notre philosophe, peut dans ces conditions donner la priorité au respect des personnes, au nom de la sollicitude qui s'adresse aux personnes dans leur singularité irremplaçable. ${ }^{24}$ En effet, elle sait que l'attitude à adopter pour chaque cas n'est pas prescrite au préalable, par une règle quelconque, d'où le fait de devoir être prudent ou attentif à la spécificité du cas.

\section{III}

Ici s'annonce tout le sens de l'effet historique de l'éthique d'Aristote, philosophe auquel Ricoeur se réfère dans ces termes: «la première grande

${ }^{21}$ P. RICOEUR (1992 : 206-207).
${ }^{22}$ P. RICOEUR (1990 : 288).
${ }^{23}$ P. RICOEUR (1990 : 311$)$.
${ }^{24}$ Cf., P. RICOEUR (1990: 305). 
leçon d'Aristote, que nous retenons, réside dans le fait qu'il ait cherché dans la praxis la racine de la 'vie bonne'. La seconde a été le fait d'avoir tenté d'ériger la téléologie interne à la praxis en principe structurant de la visée de la vie bonne ${ }^{25}$ ». "C'est chez lui qu'on trouve un discours structuré sur la praxis qui fait cruellement défaut chez Kant. Tout repose sur le concept de prohairesis, capacité de préférence raisonnable ${ }^{26}{ }_{\Downarrow}$.

C'est cette capacité de préférence que Ricoeur veut justement récupérer, même s'il ne renonce pas à Kant. D'où sa thèse fondamentale, autrement dit, le vrai nœud de la sagesse pratique: 1) en cas de conflit il faut premièrement passer du plan moral des normes au plan des convictions, de l'obligatoire à l'optatif de l'éthique (antérieure à la morale) et accepter d'agir en contexte d'incertitude; en effet, il n'y a pas un savoir déductif des choses morales. Au contraire, il y aura toujours un conflit d'interprétations. 2) Devant ce conflit et deuxièmement, la recherche du juste milieu, de la «mésotès aristotélicienne, paraît être de bon conseil. C'est que d'une manière générale les décisions morales les plus graves consistent à tirer une ligne de partage entre le permis et le défendu dans des zones elles-mêmes moyennes, résistant à des dichotomies trop familières; 3 ) troisième trait de la sagesse pratique: « l'arbitraire du jugement moral en situation est d'autant moindre que le décideur - (...) - a pris conseil des hommes et des femmes réputés, les plus compétents et les plus sages. La conviction qui scelle la décision bénéficie alors du caractère pluriel du débat. Le phronimos n'est pas forcément l'homme seul ${ }^{27}$ ».

La sagesse pratique dont Ricoeur se réclame, dans la lignée d'Aristote, a alors pour tâche principale de récupérer la pondération de la fronesis, de façon à résoudre les conflits suscités par l'application purement formelle de la règle. $\rangle^{28}$. Il lui incombe ainsi une tâche toute particulière: inventer les comportements qui satisfont au mieux l'exception qu'exige la sollicitude (pour la personne), tout en trahissant le moins possible la règle. Dans ce cadre, la transgression ou exception à la règle, en faveur de la sollicitude pour les personnes et de l'attention aux situations particulières, n'est pas, comme nous le montre dès lors le cas du procès en Droit (pour Ricoeur modèle d'une éthique appliquée), une aberration.

«Traiter l'autre simplement comme un moyen c'est, avant tout, commencer à le violenter» ${ }^{29}$. D'où le fait qu'appliquer une norme en Droit soit
25 P. RICOEUR (1990: 203).
26 P. RICOEUR (2001: 59).
27 P. RICOEUR (1990 : 317-318).
28 P. RICOEUR (1990: 305).
29 P. RICOEUR (1990: 309). 
une opération très complexe, laquelle exige une interprétation qui exclut toute logique mécanique du syllogisme pratique. En effet, la qualification elle-même d'un acte litigieux en Droit implique toujours un travail herméneutique appliqué à la situation et à la norme. Le processus qui mène à ce qu'un cas soit placé sous une norme suppose, avant tout, deux moments herméneutiques absolument reliés: A) d'un côté, le mouvement de croisement des intrigues ou histoires vraisemblables qui sont à la base de la configuration du cas. Nous savons d'ailleurs, par le débat même des tribunaux, comme il est compliqué d'extraire un récit absolument vrai de l'opposition des récits proposés par les parties en litige. B) de l'autre, du côté de la norme, il n'y a pas non plus un sens univoque, et cela veut dire qu'il n'est pas toujours clair comment savoir quelle est la norme qui s'applique à chaque cas.

L'application suppose toujours une double herméneutique: celle des faits et celle des normes (...), et le bon sens en situation apparaît au croisement de ces interprétations ${ }^{30}$. Argumentation et interprétation tissent d'ailleurs le processus qui conduit en Droit à la décision. Et ne l'oublions pas: l'idée de norme (loi) ne s'efface pas dans le bon sens en situation, au contraire, elle est enrichie et explicitée, en ce qui concerne ses contours peu clairs. On peut dire donc que jamais la sagesse pratique ne consisterait à transformer en règle l'exception à la règle.

La sagesse pratique cultivée par le Droit se propose donc comme tâche une interprétation et une argumentation qui vise le raisonnable, la délibération problématique, celle qui fait penser. Sa méthode, que Ricoeur estime bien, est d'un bout à l'autre herméneutique et elle a comme rôle principal celui d'établir des médiations, de construire l'analogue, en somme de traduire, de façon à proposer une décision. Elle est une application sous l'égide de la phronesis aristotélicienne. Et le niveau de conscience qui agit dans cette sagesse, constitutive du cadre de toutes les éthiques appliquées, est selon Ricoeur, celui de la conviction intime qui, après la méditation profonde, "habite l'âme du juge ou du jury prononçant le jugement en équité» ${ }^{31}$. Autrement dit, apparaît ici une sollicitude critique qui a déjà traversé la double épreuve des conditions morales du respect pour la norme et des conflits suscités par un tel respect. Face au tragique de l'action, la sagesse pratique sait qu'elle peut seulement toujours dire le mieux ou le moins mauvais, celui qui résulte d'un débat où les normes n'ont pas eu plus de poids que la sollicitude envers la personne. La sagesse pratique, consacrée par la création de nouvelles décisions face à des cas

30 P. RICOEUR (1995: 218).

31 P. RICOEUR (1995: 218). 
difficiles, «élabore toujours des compromis fragiles au cours desquels on essaye de choisir bien moins entre le bien et le mal, entre le blanc et le noir qu'entre le gris et le gris, ou, dans le cas hautement tragique, entre le mal et le pire $»^{32}$.

Tel est donc le mode dont Ricoeur s'approprie, de mode critique, le double héritage d'Aristote et de Kant, en essayant de nous montrer que, sans la référence à la norme, en tant que point fixe et noyau dur de l'éthique, noyau qui implique la position d'un sujet obligé à l'obligation, la conscience ne laisse jamais son dangereux narcissisme et est incapable de mener une vie éthique authentique; mais que, d'autre part, la norme considérée d'un point de vue purement formel et contraignant est à l'origine de fondamentalismes tragiques comme celui d'Antigone. Donc, la norme doit apparaître comme «règne intermédiaire entre l'éthique antérieure et l'éthique postérieure» ${ }^{33}$.

Ce qu'il faudra donc bien comprendre, c'est, d'après Ricoeur, le lien fort que la tradition scolaire a occulté et qui peut ainsi joindre la phronesis de « l'Éthique à Nicomaque et le vœu de 'vivre bien' qui la couronne, au concept de bonne volonté des Fondements de la métaphysique des mours et à celui de respect de la Critique de la raison pratique» ${ }^{34}$. Ce lien notre philosophe le découvre avec sa phénoménologie de l'homme capable. Développée dans Soi-même comme un autre, elle a pour tâche de préparer «le terrain pour cette capacité proprement éthique, l'imputabilité, capacité à se reconnaître comme l'auteur véritable de ses actes». ${ }^{35}$ En effet l'idée d'imputabilité peut être tour à tour associée au concept grec de préférence raisonnable et à l'idée kantienne d'obligation morale. Mais c'est dans les éthiques appliquées, telles que le droit et l'éthique médicale que Ricoeur voit la vertu de la prudence être mise à l'épreuve de la pratique. Des deux côtés il s'agit de passer des normes et des connaissances théoriques sur les normes à une décision concrète en situation. Et c'est toujours dans le jugement singulier que cette application s'opère.

Terminons donc avec un texte où le philosophe dit son projet éthique, à partir d'un point de vue anthropologique: «Je tiens beaucoup à cette avancée à partir d'un premier niveau où l'on répond à la question aristotélicienne: Que signifie la poursuite de la vie bonne? vers un second niveau où l'on répond à la question kantienne: Qu'est ce qu'obéir au

\footnotetext{
32 P. RICOEUR (1995: 220).

33 P. RICOEUR (2001: 56).

${ }^{34}$ P. RICOEUR (2001: 62).

35 P. RICOEUR (2001: 65).
} 
devoir? pour parvenir à un troisième niveau où l'on se demande: qu'est ce que résoudre un problème éthico-pratique inédit ? - c'est le problème de la sagesse pratique, que je rattache à l'herméneutique de l'application', sous l'égide de la phronesis aristotélicienne ${ }^{36_{\text {» }}}$.

\section{BIBLIOGRAPHIE}

P. RICOEUR, Philosophie da la volonté. Finitude et culpabilité. I L'homme faillible, Paris, Aubier, 1960.

P. RICOEUR, Soi-même comme un autre, Paris, Seuil, 1990.

P. RICOEUR, Lectures 2. La contrée des philosophes, Paris, Seuil,1992.

P. RICOEUR, Le juste, Paris, Esprit, 1995.

P. RICOEUR, La critique et la conviction. Entretien avec François Azouvi et Marc de Launay, Paris, Calmann-Lévy, 1995.

${ }^{36}$ P. RICOEUR, (1995 : 141-142). 